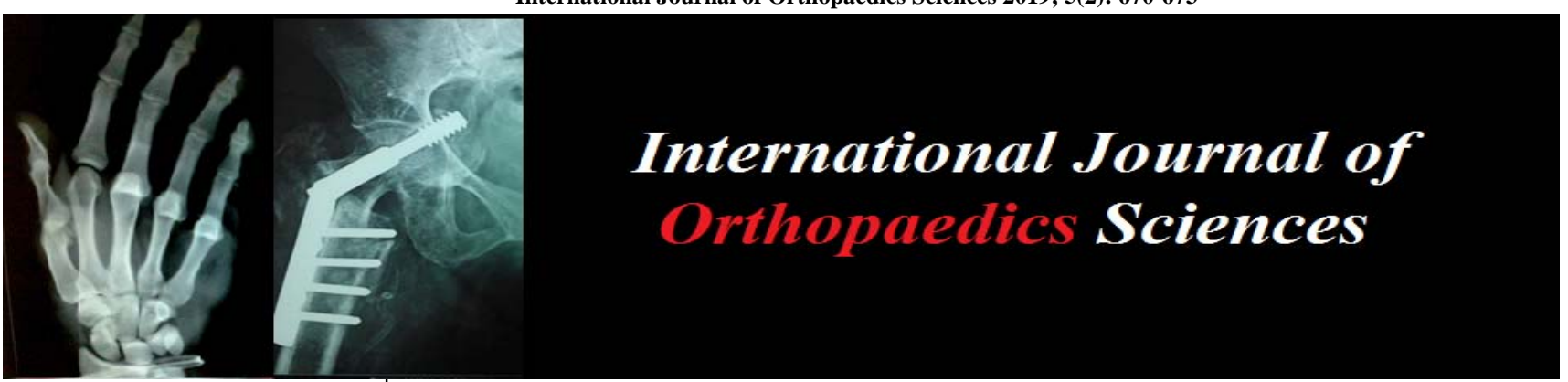

ISSN: $2395-1958$

IJOS 2019; 5(2): 670-673

(C) 2019 IJOS

www.orthopaper.com

Received: 21-02-2019

Accepted: 23-03-2019

Dr. K Mohan Kumar

Professor, Department Of

Orthopaedics, Govt. Stanley

Medical College, Chennai,

Tamil Nadu, India

Dr. Parthasarathy $\mathbf{S}$

Major, Junior Resident,

Department of Orthopaedics,

Govt. Stanley Medical College,

Chennai, Tamil Nadu, India

Dr. Varrdhaman Dhariwal

Assistant Professor, Department of Orthopaedics, Govt. Stanley

Medical College, Chennai,

Tamil Nadu, India
Correspondence

Dr. Parthasarathy $S$

Major, Junior Resident,

Department of Orthopaedics,

Govt. Stanley Medical College,

Chennai, Tamil Nadu, India

\section{Secondary osteoarthritis hip in young individuals managed with primary uncemented total hip replacement using ha-coated conventional stem and acetabular component}

\author{
Dr. K Mohan Kumar, Dr. Parthasarathy S and Dr. Varrdhaman \\ Dhariwal
}

DOI: $\underline{\text { https://doi.org/10.22271/ortho.2019.v5.i2i.70 }}$

\section{Abstract}

Background: The management of young individuals seeking medical attention due to progressive hip pain as a result of secondary osteoarthritis and who want active and pain-free hip was challenging in past. The actual incidenece of secondary osteoarthritis is difficult to ascertain. Young individuals with secondary osteoarthritis hip due to developmental dysplasia of hip, coxa plana (Legg-Calvé-Perthes disease), post-traumatic, slipped capital femoral epiphysis, Paget disease, Haemophilia, Avascular necrosis of femoral head, and so on can be effectively managed with primary uncemented total hip replacement (THR) with good functional outcome in short-term follow-up. This will increase the revision THR rate later. Long-term follow-up is required to watch for prosthesis-related complications

Materials and Methods: Material includes 64 patients who attended our OPD from Oct 2013 to Dec2017 and all of them were diagnosed to have secondary osteoarthritis hip with poor hip function as assessed by Harris hip score. All of them underwent uncemented total hip replacement (THR) through posterior/anterolateral approach to hip. Conventional HA-coated stem and acetabular components were used. They were post-operatively assessed at regular intervals with Harris hip score and were found to have excellent functional outcome with high level of patient satisfaction.

Result: All patients have excellent Harris hip scorepost-operatively. Patients became independent and were able to carry out day-to-day activities with ease. No complications were noticed. The dramatic change in their hip function was appreciable even on the day of surgery itself.

Conclusion: We conclude that uncemented THR using conventional HA-coated components is an excellent surgical modality for the management of secondary osteoarthritis in young individuals with poor hip function. The functional outcome is promising and makes patient independent and pain free. The said procedure is cost-effective and reliable based on excellent hip function achieved in immediate post-operation. Long-term follow-up is recommended.

Keywords: Secondary osteoarthritis hip, uncemented THR, conventional HA-coated stem

\section{Introduction}

The management of patients presenting with late secondary osteoarthritis, with primary uncemented THR is challenging and meticulous pre-operative planning to assess acetabular deficiency, abductor function is required ${ }^{[1,2]}$. Also patient's expectation should be considered and all should be explained about the chances of early revision and complications associated with it. Originally the primary indication for total hip arthroplasty was relief from arthritic hip pain in patients aged $>65$ years, in whom non-operative management is not helpful. After the proper evaluation, the procedure was found to be very successful and indication extended to other conditions too. The 1994 National Institute of Health Consensus Statement on Total Hip Replacement concluded that "THR [total hip replacement] is an option for nearly all patients with diseases of the hip that cause chronic discomfort and significant functional impairment." Charnley warned that (1) the procedures suitable for older patients may not be suitable for younger patients; (2) the problems in bilateral disease are different from the problems in unilateral disease; (3) the treatment for an arthritic hip with good range of motion is different from the treatment for an arthritic hip with poor range of motion; and (4) the demands on the 
hip in a labourer are greater than the demands in a sedentary worker. The potential for failure in young patients and the increased risk of infection and other complications that makes revision of the procedure become necessary must be emphasized. Pain in the presence of a degenerative or destructive process in the hip joint as evidenced on imaging studies is the primary indication for surgery.

The important causes for secondary osteoarthritis hip are secondary developmental dysplasia of hip, coxa plana (LeggCalvé-Perthes disease), post-traumatic, slipped capital femoral epiphysis, Paget disease, AVN and Haemophilia ${ }^{[3]}$. The routine procedure has to be modified for specific disorder to achieve better results. Careful removal of osteophytes is necessary and acetabular evaluation pre-operatively and intraoperatively should be thorough. Proper patient counselling and selection is crucial. Froberg et al. found that $13 \%$ of patients with coxa plana eventually required hip arthroplasty; the prevalence was highest in patients with more severe head involvement. The incidence of primary THR in young individuals aged $<50$ years is on rise and complications associated has to be tackled with proper patient selection and accurate pre-operative planning.

Our emphasis here is on use of conventional HA-coated stem and acetabular component without using modular prosthesis, most of secondary osteoarthritis cases can be managed if preoperative assessment and planning are good. The osteointegration is good and it is cost-effective ${ }^{[4,5]}$. The patient satisfaction achieved is exemplary and no complications noted in short-term follow-up ${ }^{[6]}$.

\section{Materials and Methods}

We have done study of patients $<50$ years of age who were diagnosed to have secondary osteoarthritis hip and managed with uncemented THR with HA-coated conventional stem and acetabular component from Oct 2013 to Dec 2017.Our study comprised of 64 patients of which 44 were male and 20 were female.Most of them were daily wagers and from poor economic status.As most of the patients were young, comorbidities were less and only 4 male patients were diabetic with good glycaemic control. Maximum age was 49 and minimum 17, both were males. Average age was 35.9 years with male average 36.5 and female average 34.5 years. Most patients' symptoms were chronic in nature with pain most disturbing symptom. Patients were found to have flexion, adduction and external rotation deformity with many having shortening $>1 \mathrm{~cm}$.No neurovascular deficit was present in any of the cases. Diagnosis of secondary arthritis is made on basis of history, clinical examination and plain radiograph pelvis with both hips anteroposterior and Frog leg lateral or cross table lateral view. CT was taken to assess the osteophyte extent and to delineate native bone so that cup placement can be accurate. MRI was taken in patients suspected to have avascular necrosis of head. Pre-operatively CRP, ESR, urine culture is obtained and septic foci in ear nose throat, skin, tooth were ruled out to avoid post-operative infection. If infection is found to be present, a course of antibiotics is given followed by surgery. All patients' Harris hip score was assessed pre-operatively.

Out of 64 patients, AVN was present in 47 out of which bilateral cases were 15 ,Perthe's disease ${ }^{[7]}$ in 4 , SCFE-related osteoarthritis in 1, post-traumatic in 4 and post-traumatic fixation failure related osteoarthritis in $8^{[8,9,10]}$. In bilateral AVN cases, staged surgery was done. All cases showed male predominance except in Perthe's disease ${ }^{[11]}$.

\begin{tabular}{|c|c|c|c|c|}
\hline Diagnosis & Male & Female & Total & Percentage \\
\hline B/L AVN & 12 & 3 & 15 & 23.43 \\
\hline AVN & 21 & 11 & 32 & 50 \\
\hline Perthe's disease & 1 & 3 & 4 & 6.25 \\
\hline SCFE & 1 & 0 & 1 & 1.57 \\
\hline Post-traumatic & 4 & 0 & 4 & 6.25 \\
\hline Post-traumatic fixation failure & 5 & 3 & 8 & 12.5 \\
\hline
\end{tabular}

The procedure done was uncemented THR with conventional HA-coated stem and acetabular component and polyethylene liner. Screw fixation of acetabular component was used in all cases. Posterior approach and anterolateral approaches were used [5], anterolateral in 12 patients and rest posterior approach. Thorough soft tissue was advocated in all approaches. Most commonly used stem size was size '1' and acetabular cup size was $52 \mathrm{~mm}$. $28 \mathrm{~mm}$ metal head and polyethylene liner of appropriate size was used.

\begin{tabular}{|c|c|c|c|}
\hline Stem size & Male & Female & Total \\
\hline 0 & 6 & 7 & 13 \\
\hline 01 & 1 & 0 & 1 \\
\hline 1 & 19 & 10 & 29 \\
\hline 2 & 11 & 3 & 14 \\
\hline 3 & 5 & 0 & 5 \\
\hline 4 & 1 & 0 & 1 \\
\hline 5 & 1 & 0 & 1 \\
\hline
\end{tabular}

\begin{tabular}{|c|c|c|c|}
\hline Acetabular cup size & Male & Female & Total \\
\hline 46 & 3 & 6 & 9 \\
\hline 48 & 4 & 4 & 8 \\
\hline 50 & 7 & 5 & 12 \\
\hline 52 & 15 & 4 & 19 \\
\hline 54 & 11 & 0 & 11 \\
\hline 56 & 2 & 1 & 3 \\
\hline 58 & 2 & 0 & 2 \\
\hline
\end{tabular}

Rehabilitation according to universal THR post-operative rehabilitation protocol started on the day of surgery itself. Patients were allowed to weight bear partially on next day. Post-operatively Harris hip score was used to assess the hip function at regular intervals and radiographs were taken to look for complications.

\section{Results}

The prosthesis fitting in all cases were found to be stable and adequate for mobilisation. There was no neurovascular deficit. One patient developed superficial surgical site infection which subsided after dressing and antiobiotics. Three patients have residual limp and LLD was reduced to $<1 \mathrm{~cm}$ in all patients. Abductor function was assessed to be good. Harris hip score was poor in all patients preoperatively. Post-operatively 42 showed excellent score (score between 90 and 100), 17 good (score between 80 and 89) and 5 fair (score between 70 and 79).According to Harris hip score, successful result is defined as post-operative increase of score by $>20$, radiologically stable prosthesis and no additional femoral reconstruction. Our all patients had successful result in short-term follow-up.

\section{Discussion}

Total hip replacement has earned the title of "the operation of the century." The incidence of performing THR in young individuals with secondary osteoarthritis is increasing because of promising results shown in post-operative follow-up till date. Ajay Malviay et al. in their review of registry data recommended that most joint registry data favour the use of 
cemented prosthesis in the elderly population, the definition of which can be difficult, but physiological patients over the age of 65 years will do better with cemented prosthesis with prosthetic survival meeting the NICE benchmark of $>95 \%$ survival at 10 years. For the younger population, uncemented and hybrids are certainly good options to be considered to improve survival, but the results may not meet the NICE benchmark in patients younger than 50 years. We have used HA-coated polar stem and polar cup which is meeting NICE recommendation. Takenaga et al. concluded that cementless total hip arthroplasty with use of a second-generation, extensively coated stem demonstrated durable fixation in an active, younger population at a minimum follow-up of 10 years and had better survivorship with respect to fixation compared with their previously reported cemented cohort. Uncemented THR is the choice of replacement in young individuals. Adelani MA et al. concluded that in THR in young although clinical outcome scores remained constant, aseptic loosening and revision rates decreased substantially with more contemporary procedures.

Babovic $\mathrm{N}$ et al. concluded that $36 \mathrm{~mm}$ metal head coupling with HXLPE as the good alternate articulation surface when planning THA for patients aged 50 years and less. Dumbleton $\mathrm{J}$ in his study found that the clinical results at 15 years after total hip replacements have demonstrated that hydroxyapatitecoated femoral stems perform as well as, and possibly better than, other types of cementless devices, with the added benefit of providing a seal against wear debris. Hydroxyapatite-coated acetabular components must have a mechanical interlock with bone in order to take advantage of the coating effects ${ }^{[3]}$. Clinical analyses of these types of designs at seven years have indicated good survivorship. Know MS demonstrated that adequate soft tissue repair greatly reduces the relative risk of dislocation using the posterior approach, and that the dislocation rates for the three most prevalent approaches (anterolateral, direct lateral and posterior) are similar. We have used posterior approach and anterolateral approach ${ }^{[5]}$.

In secondary osteoarthritis, the extremity is often shortened and more if there is head or acetabulum deformation. The hip is usually flexed, externally rotated and adducted. During surgery, the osteophytes may block dislocation of head and anterior/posterior osteophytes should be removed thoroughly. The acetabular subchondral bone will be thick and extra reaming is required to get bleeding surface for bone ingrowth. Osteophyte in the pulvinar may obscure the medial wall. Care should be taken so that the acetabular cup is not supported by osteophytes rather than native bone. Greater trochanter may be enlarged and some part may require removal to avoid impingement. The anatomic abnormalities associated with other disorders should be considered in pre-operative planning.

Pre-operative evaluation should rule out any major systemic debilitating disease, infection and systemic disease that can be a contraindication for surgery. The radiographic evaluation for acetabular and femoral deformation should be meticulously done. Most of patients with secondary osteoarthritis have a long history and the chances of deficiency are high. Displacement of head, medialisation of head, acetabular breech/deficiency, LLD, osteophyte's position, head size, stem size, neck cut required and vertical/horizontal offsets all should be well planned. Any pathology of other joints should be assessed and basic lab investigation must include serum ALP because Paget's disease is one of cause for secondary osteoarthritis hip.
The THR for severely functionally affected hip is costeffective as the functional outcome is excellent in short-term follow-up. As all THR is performed in young individuals in our study, the rates of revision is expected to be high. Between 1990 and 2002, 17.5\% of all hip arthro plasties performed in the United States were revision procedures. XLPE liners have significantly reduced wear and are associated with a greater implant survival rate at 10 years compared with conventional UHMWPE liners. The revision surgeries are always complex and associated with blood loss, infection, fractures, dislocations and nerve injuries and it emphasize the need for precise primary procedure. The most common type of revision total hip arthroplasty procedure performed was all-component revision (41.1\%), and the most common causes of revision were instability/dislocation (22.5\%), mechanical loosening (19.7\%) and infection (14.8\%) according to study by Bozic et al. Springer BD in his study found that the most common reasons for failure were instability (49 of 141 hips, 35\%), aseptic loosening (42 of 141 hips, 30\%), osteolysis and/or wear (17 of 141 hips, 12\%), infection (17 of 141 hips, 12\%), miscellaneous (13 of 141 hips, 9\%) and periprosthetic fracture (three of 141 hips, 2\%). Survivorship for revision total hip arthroplasty using second revision as endpoint was $82 \%$ at 10 years. Aseptic loosening and instability accounted for $65 \%$ of these failures. Regular follow-up is required for early detection of complications in young individuals undergoing THR. Pellicci PM et al. concluded that there is an increased failure rate with longer follow-up of revision total hip replacement, and that progressive radiolucency at an interface indicates a poor prognosis for the arthroplasty. So follow-up visits planned and radiographs taken at 6 weeks, 3 months, 1 year and periodically thereafter every 2 years and radiographs compared with previous one to rule out loosening, migration, wear, implant failure because most of these can occur without clinical symptoms and makes revision difficult if detected late.

\section{Conclusion}

We conclude that primary uncemented THR using conventional HA-coated components is a versatile and costeffective procedure recommended for painful secondary arthritis hip with poor function. The post-operative functional outcome is relatively accurately predictable that it will be excellent in most of the patients without any co-morbities ${ }^{[12,}$ 13, 14]. The complications of THR in young individuals are associated with acetabular and femoral changes as a result of primary disease in primary THR and revision THR later. Appropriate pre-operative planning, patient counselling and patient selection are all key points in the successful hip function for a reasonable time till revision is required. The surgeon and patient should be prepared for the revision surgery in long-term follow-up.

\section{Financial support and sponsorship}

Nil.

\section{Conflicts of interest}

There are no conflicts of interest.

\section{References}

1. Takenaga RK, Callaghan JJ, Bedard NA et al. Cementless total hip arthroplasty in patients fifty years of age or younger: a minimum ten-year follow-up, J Bone Joint Surg. 2012; 94:21-53. 
2. Adelani MA, Keeney JA, Palisch A et al. Has total hip arthroplasty in patients 30 years or younger improved? A systematic review, Clin Orthop Relat Res. 2013; 471:2595.

3. Babovic N, Trousdale RT. Total hip arthroplasty using highly cross-linked polyethylene in patients younger than 50 years with minimum 10-year follow-up, J Arthroplasty. 2013; 28:815.

4. Dumbleton J, Manley MT. Current concepts review: hydroxyapatite-coated prostheses in total hip and knee arthroplasty, J Bone Joint Surg. 2006; 86A:25-27.

5. Kwon MS, Kuskowski M, Mulhall KJ et al. Does surgical approach affect total hip arthroplasty dislocation rates? Clin Orthop Relat Res. 2006; 447:34.

6. Al-Khateeb H, Kwok IHY, Hanna SA et al. Custom cementless THA in patients with Legg-Calve-Perthes disease, J Arthroplasty. 2014; 29:792.

7. Baghdadi YMK, Larson AN, Stans AA, Mabry TM. Total hip arthroplasty for the sequelae of Legg-CalvéPerthes disease, Clin Orthop Relat Res. 2013; 471:29-80.

8. Makridis KG, Obakponovwe O, Bobak P, Giannoudis PV. Total hip arthroplasty after acetabular fracture: incidence of complications, reoperation rates and functional outcomes: evidence today, J Arthroplasty. 2014; 29:19-83.

9. Hio AM, Van Grinsven S, Lucas C et al. Partial versus unrestricted weight bearing after an uncemented femoral stem in total hip arthroplasty: recommendation of a concise rehabilitation protocol from a systematic review of the literature, Arch Orthop Trauma Surg. 2010; 130:547.

10. Z Rheumatol, 2017. doi: 10.1007/s00393-017-0408-z. [Epub ahead of print] [Indications for joint replacement: Total hip arthroplasty]

11. Zhongguo Gu Shang. [Middle and long-term results of total hip arthroplasties for secondary post-traumatic arthritis and femoral head necrosis after acetabular fractures].Yu L, Zhang $\mathrm{CH}$, Guo T, Ding H, Zhao JN, 2016; 29(2):109-13.

12. Acta Orthop Traumatol Turc. [Total hip arthroplasty in patients with osteoarthritis secondary to developmental dysplasia of the hip: results after a mean of eight-year follow-up]. Bektaşer B1, Solak S, Oğuz T, Oçgüder A, Akkurt MO, 2007; 41(2):108-12.

13. Chir Narzadow Ruchu Ortop Pol. [Total hip replacement after Legg-Calvé-Perthes disease]. Pietrzak K1, Strzyzewski W, Pucher A, Kaczmarek W, 2011; 76(3):129-33.

14. J Bone Joint Surg Am. doi: 10.2106/JBJS.J.00648. Longterm results of total hip replacement in patients with Legg-Calvé-Perthes disease. Traina F1, De Fine M, Sudanese A, Calderoni PP, Tassinari E, Toni A, 2011; 93(7):e25. 